\title{
Could surgical management improve the IVF outcomes in infertile women with endometrioma?: a review
}

\author{
Hyun Jong Park', Hannah Kim², Geun Ho Lee², Tae Ki Yoon³, Woo Sik Lee ${ }^{3}$ \\ ${ }^{1}$ Fertility Center of CHA Gumi Medical Center, ${ }^{2}$ Department of Obstetrics and Gynecology, CHA Gumi Medical Center, CHA University School of \\ Medicine, Gumi; ${ }^{3}$ Department of Obstetrics and Gynecology, Fertility Center of CHA Gangnam Medical Center, CHA University School of Medicine, \\ Seoul, Korea
}

Endometriosis is a chronic inflammatory condition that affects fertility and could be toxic to the ovary. Endometrioma per se and surgical interventions for endometrioma significantly reduce the ovarian reserve. Therefore, to prepare for surgical intervention for endometrioma, the high-risk group with decreased ovarian reserve must be considered. There is no evidence to support the use of surgical intervention before in vitro fertilization (IVF) to improve the reproductive outcomes of subsequent IVF in infertile women with advanced-stage endometriosis or endometrioma. As surgical treatment has few benefits, IVF could be recommended immediately for aiding conception in these women. However, the reproductive prognosis of IVF may be worse in the more advanced stages of endometriosis. When dysmenorrhea is severe or when cancer is suspected, surgery prior to IVF may be necessary and justified. When the size of the endometrioma is very large, surgery could be required prior to IVF to facilitate access to follicles during oocyte retrieval or to improve the ovarian response to controlled ovarian stimulation. Prolonged pituitary downregulation in women with surgically diagnosed endometriosis may be helpful to increase the clinical pregnancy rate in subsequent IVF cycles. The purpose of this paper was to review the efficiency and clinical application of the surgical intervention and IVF for infertile women with advanced-stage endometriosis or endometrioma.

Keywords: Endometriosis; In vitro fertilization; Laparoscopy; Cystectomy; Infertility

\section{Introduction}

Endometriosis is a chronic inflammatory condition that affects fertility. The prevalence rate of endometriosis in infertile women is estimated to be $25-40 \%$ [1]. It is responsible for approximately $10 \%$ of the indications for in vitro fertilization (IVF) [2].

Endometriosis affects fertility by making the in vivo environment harmful for an oocyte or an embryo. Theoretically, surgical treatment of endometriosis could create a more favorable environment for successful conception [3]. On the other hand, surgical intervention for endometrioma may increase the risk of infertility by reducing the ovarian reserve [4].

The spontaneous pregnancy rate at 8 months after surgery was reported to be approximately $30 \%$ in infertile patients with minimal/mild endometriosis [5]. This figure is similar to the clinical pregnancy rate following a single IVF trial. In addition, as the endometriosis stage is advanced, the spontaneous pregnancy rate in expectant management may be reduced further [4]. Therefore, the IVF procedure in patients with endometriosis could be justified from the costeffectiveness aspect. Clinicians are usually concerned about performing an operation for advanced-stage endometriosis or endometrioma before attempting an IVF $[6,7]$.

The purpose of this paper is to review the efficiency and clinical application of the surgical intervention and IVF in infertile women with advanced-stage endometriosis or en-

Received: 2018.05.28. Revised: 2018.08.17. Accepted: 2018.08.21. Corresponding author: Woo Sik Lee

Department of Obstetrics and Gynecology, Fertility Center of CHA Gangnam Medical Center, CHA University School of Medicine, 569 Nonhyeon-ro, Gangnam-gu, Seoul 06125, Korea

E-mail: wooslee@cha.ac.kr

https://orcid.org/0000-0002-2329-1774

Articles published in Obstet Gynecol Sci are open-access, distributed under the terms of the Creative Commons Attribution Non-Commercial License (http://creativecommons. org/licenses/by-nc/3.0/) which permits unrestricted non-commercial use, distribution, and reproduction in any medium, provided the original work is properly cited.

Copyright $\odot 2019$ Korean Society of Obstetrics and Gynecology 


\title{
Obstetrics \& Gynecology Science
}

\author{
Vol. 62, No. 1, 2019
}

dometrioma, on the basis of the results of recent clinical research studies, reviews, and meta-analyses. The views on spontaneous pregnancy in patients with advanced-stage endometriosis was excluded from the focus of this review. The presence of endometrioma was considered as advancedstage endometriosis based on the revised American Society for Reproductive Medicine classification of endometriosis [8]. Published studies were searched on PubMed and Google Scholar, combining the key terms "endometriosis" and/or "endometrioma" and/or "in vitro fertilization (IVF)" and/or "infertility" and/or "fertility" and/or "laparoscopy" and/or "cystectomy" and/or "surgery" on December 31, 2017. The main inclusion criteria of study subjects for our review were the IVF outcomes of patients with endometrioma and/or advanced-stage endometriosis and/or following surgical intervention for endometrioma. Studies published in languages other than English and those without original full text articles were also excluded from our review. In our review, we considered intracytoplasmic sperm injection cycles as IVF cycles for convenience.

\section{Toxic effects of endometrioma on the ovary}

The molecular mechanisms by which endometrioma causes ovarian toxicity are summarized as follows.

First, endometriotic cysts contain high levels of cell damage-mediating factors such as proteolytic enzymes, inflammatory mediators, reactive oxygen species (ROS), and iron [9-11]. Thus, endometrioma per se exerts a toxic effect on healthy tissues around the cyst. These toxic contents may interfere with the expression of critical genes in the cells surrounding the endometriotic cysts, resulting in the disruption of normal folliculogenesis and causing subsequent degradation of oocytes [11,12].

Second, when endometriosis spreads to the ovaries, oxidative stress levels increase in the ovarian cortex and oocyte apoptosis may occur, causing necrosis of early follicles and a consequent reduction in follicular density. In addition, high levels of ROS suppress ovarian angiogenesis, and ovarian interstitial microvascular injury takes place, which leads to a reduction in blood perfusion to the ovarian cortex [13-16]. Ultimately, the ovarian reserve may be reduced by an endometrioma, which adversely affects pregnancy outcomes.
Simón et al. [17] analyzed IVF cases with donated oocytes and reported that the pregnancy rate was significantly reduced when IVF was performed using oocytes from patients with endometriosis, regardless of the condition of the recipients. Díaz et al. [18] also reported that the IVF pregnancy rate was significantly lower in recipients who received oocytes from oocyte donors with endometriosis than in those who received oocytes from donors without endometriosis. According to a prospective study by Shebl et al. [19], the occurrence rates of metaphase II (MII) oocytes and morphologically normal oocytes were reported to be significantly lower in the endometriosis group than in the non-endometriosis group. Endometriosis stage IV was significantly associated with worse-quality oocytes than stages I-III. Thus, it may be reasonable to judge that endometriosis is clinically likely to reduce the quality of oocytes.

\section{Meta-analyses of IVF outcomes in women with advanced-stage endometriosis}

Barnhart et al. [20] reported in their meta-analysis that IVF outcomes worsened as endometriotic disease became more advanced. When comparing the IVF pregnancy rate in infertility patients with a tubal factor, the reduction in the IVF pregnancy rate was approximately $20 \%$ in patients with mild endometriosis, while the reduction was $>50 \%$ in patients with extensive/severe endometriosis.

In 2 published meta-analyses, the IVF outcomes in patients with minimal/mild endometriosis were similar to those in patients for whom IVF was performed for other indications, while the outcomes were inferior in infertile patients with moderate/severe endometriosis (fewer oocytes retrieved, lower implantation rate, and lower birth rate) [21,22]. Harb et al. [21] reported that in cases with stage III/IV endometriosis, both the implantation and clinical pregnancy rates were significantly reduced by $21 \%$.

Yang et al. [23] recently conducted a meta-analysis that limited cases to patients with endometrioma. They reported that while fewer oocytes and MII oocytes were retrieved and fewer embryos were formed in patients with endometrioma than in the controls, no significant difference was found between the patient and control groups in terms of clinical pregnancy and live birth rates. This result did not correspond 


\section{Obstetrics \& Gynecology Science}

Hyun Jong Park, et al. Surgery and IVF in endometrioma

with the results of the two above-mentioned meta-analyses $[21,22]$. However, in the meta-analysis of Yang et al. [23], all of the patients who underwent surgery for endometrioma were excluded. As the study subjects were restricted to patients with relatively small-sized endometriomas, selection bias was highly possible. In addition, the few number of involved observational studies was the limitation (three or fewer studies) of the meta-analysis for clinical pregnancy and live birth rates. An inverse relationship was observed; that is, the larger the endometrioma size, the fewer the number of follicles in the ovary, which suggests that the size of the endometrioma itself could be an indicator of the aggressiveness of the disease [24]. The meta-analysis of Yang et al. [23] should be reconsidered given that endometrioma per se negatively affects ovarian reserve even before surgery [25] and that several molecular mechanisms suggest direct toxic effects of endometrioma on ovarian parenchyma and folliculogenesis.

From a clinical perspective, it is reasonable to conclude that the reproductive prognosis of IVF would be worse in the more advanced stage of endometriosis and with more increased endometrioma size.

\section{Surgical intervention for endometrioma in infertility management}

The dominant opinion is that surgical intervention for endometrioma significantly reduces the ovarian reserve as represented by decreased serum AMH levels [26-28]. According to the meta-analysis of Raffi et al. [28], serum AMH levels were reduced by approximately $40 \%$ after laparoscopic excision of endometrioma. Endometrioma per se and surgical interventions for endometrioma reduced the ovarian reserve [26,28-30]. Surgical intervention is likely to further reduce ovarian reserve in patients with endometrioma, whose ovarian reserve has already been reduced by endometrioma per se. In fact, according to the prospective cohort study conducted by Goodman et al. [31], baseline serum AMH levels in the endometrioma group were significantly lower than those in the control group without endometrioma, and serum AMH levels were significantly lower at 1 month after surgery. Furthermore, the larger the size of the endometrioma resection, the greater the reduction in the AMH level after surgery [32].

Roustan et al. [33] reported that the clinical pregnancy and live birth rates per IVF cycle were significantly lower in patients with decreased ovarian reserve (DOR) after surgery for endometrioma than in patients with idiopathic DOR. The retrospective study of Hong et al. [34] compared IVF outcomes between a DOR group without ovarian surgery and an endometrioma surgery-induced DOR group. They concluded that the clinical pregnancy and live birth rates were not significantly different between the two groups. However, Hong et al. [34] reported that the clinical pregnancy rate per cycle ( $8.5 \%$ vs. $20.2 \%$ ) and live birth rate per cycle (4.2\% vs. $13.4 \%)$ tended to be lower in the endometrioma surgeryinduced DOR group than in the DOR group without ovarian surgery. The critical weakness of this study was the small sample size, where only 47 IVF cycles were included in the endometrioma surgery-induced DOR group. Therefore, the lack of significant differences could be attributed to the small sample size. In a retrospective study by Maignien et al. [35] involving 359 patients diagnosed as having endometriosis who underwent IVF cycles, multivariate logistic regression analysis was conducted to identify the prognostic factors that affected pregnancy rates. The results suggested that a history of surgery for endometrioma (odds ratio [OR], 0.39; 95\% confidence ratio $[\mathrm{Cl}], 0.18-0.84)$, anti-Müllerian hormone (AMH) levels of $<2 \mathrm{ng} / \mathrm{mL}(\mathrm{OR}, 0.51 ; 95 \% \mathrm{Cl}, 0.28-0.91)$, and antral follicle count (AFC) of $<10(\mathrm{OR}, 0.27 ; 95 \% \mathrm{Cl}$, $0.14-0.53)$ had adverse effects on assisted reproductive technology (ART) outcomes. Therefore, to prepare for surgical intervention for endometrioma, the high-risk patient group with DOR should be considered on the basis of the following factors [3]. First, poor ovarian responders should be identified by monitoring ovarian reserve markers such as baseline serum AMH level before surgery. Second, another consideration involves the patient's age and presence of bilateral lesions. According to a prospective study by Alborzi et al. [36], serum AMH levels after laparoscopic cystectomy of endometriomas were significantly reduced in women aged $>38$ years and diagnosed as having bilateral endometriomas. Third, the repeat surgery for recurrent endometriomas may lower $\mathrm{AMH}$ and AFC levels, and increase basal follicle-stimulating hormone (FSH) levels, resulting in a detrimental effect on the ovarian reserve $[37,38]$. According to a retrospective study by Park et al. [39], a comparison of patients who underwent IVF cycles without the second-line surgery for recurrent endometrioma with those who underwent IVF cycles after the surgery showed significantly higher deleterious effects of 


\section{Obstetrics \& Gynecology Science}

Vol. 62, No. 1, 2019

second-line surgery on ovarian response, and implantation and clinical pregnancy rates during IVF cycles. Owing to the risk of premature ovarian failure after surgery for endometriomas in the above-mentioned cases, preoperative banking of oocytes and embryos could be options to preserve fertility before surgery.

In terms of IVF outcomes, the benefit of surgical intervention for endometrioma is uncertain. Xing et al. [40] reported in a recent retrospective study that the number of MII oocytes collected during IVF cycles was significantly lower in women who had previously undergone a laparoscopic endometrioma cystectomy than in women with pelvic endometriosis. In three recently published meta-analyses that compared patients with and patients without surgery for endometrioma, the numbers of total oocytes and MII oocytes collected during IVF cycles were significantly lower in the surgery group than in the no surgery group, but no significant differences were observed between the two groups in terms of live birth and clinical pregnancy rates $[23,41,42]$. The results suggested that surgical intervention for endometrioma failed to improve reproductive outcomes in IVF cycles (Table 1). In addition to meta-analyses, several review articles consistently suggested no evidence of improvement in reproductive outcomes of IVF after surgical intervention in patients with endometrioma prior to IVF cycles [6,43-45].

In cases of infertility associated with advanced-stage endometriosis, including endometrioma, IVF could be recommended immediately to achieve pregnancy, as surgical treatment has few benefits [46]. However, surgery for severe endometriosis, including endometrioma, may be considered before IVF in the following cases: first, when the pain related to endometriosis is severe [3] and, second, when malignancy cannot be excluded [7]. Third, according the 2014 European Society of Human Reproduction and Embryology guidelines, for patients with endometrioma measuring $\geq 3 \mathrm{~cm}$, laparoscopic ovarian cystectomy is recommended before IVF to reduce the risk of infection during oocyte retrieval and facilitate access to follicles, or to improve the ovarian response to controlled ovarian stimulation [4]. Fourth, surgical intervention could be considered when the qualities of the oocytes and embryos were found to be poor in IVF cycles before surgery, or in cases of recurrent implantation failure. Theoretically, surgery for endometrioma could facilitate follicular development by reducing the tension in the ovarian tissue. Furthermore, it could reduce the levels of inflammatory factors in follicular fluid $[47,48]$. Soriano et al. [49] performed extensive laparoscopic excisional surgery, including bowel and urinary resection, in 78 patients with severe endometriosis who had repeated IVF failures and reported that 33 patients (42.3\%) conceived successfully after surgery (30 patients conceived via IVF cycles, while 3 cases conceived naturally). Restoration of a normal uterine anatomy by surgical intervention could help improve IVF outcomes in these women. However, endometrioma per se and surgical intervention could have toxic effects on ovarian follicles [50]. Consideration of the ovarian reserve may be essential before surgery for severe endometriosis, including endometrioma.

\section{Prolonged pituitary downregulation prior to IVF}

In cases of infertile women with endometriosis, the treatment approach prior to IVF must be individualized. According to the study conducted by Sallam et al. [51] that metaanalyzed three RCTs in women with surgically diagnosed endometriosis and compared the study group that received gonadotrophin-releasing hormone $(\mathrm{GnRH})$ agonist treatment for 3 to 6 months before IVF with the control group that received no treatment prior to IVF, the clinical pregnancy rate was significantly improved by 4.28 -fold in the study group as compared with the control group. The GnRH agonist could lower concentrations of various inflammatory cytokines such as IL-6 [52-54]. As a result, this can reduce the toxic effects of peritoneal cytokines on oocytes or embryos. Prolonged pituitary downregulation prior to IVF could be helpful for infertile women with endometriosis.

On the other hand, long-term administration of the GnRH agonists could suppress the expression of implantation factors, which could lead to decreased endometrial receptivity [55]. In addition, use of the GnRH agonist could induce side effects such as hot flashes, vaginal dryness, and decreased bone mineral density. Recently, as an alternative for reducing the side effects of long-term GnRH agonist treatment, dienogest, a novel progestin, is gaining attention [56]. Muller et al. [57] conducted a prospective cohort study that compared pregnancy rates in the following 3 groups after surgical intervention for endometriosis: a group of patients who took $2 \mathrm{mg}$ of dienogest daily for 6 months prior to IVF (group I), a group of patients who had monthly GnRH agonist treatment 


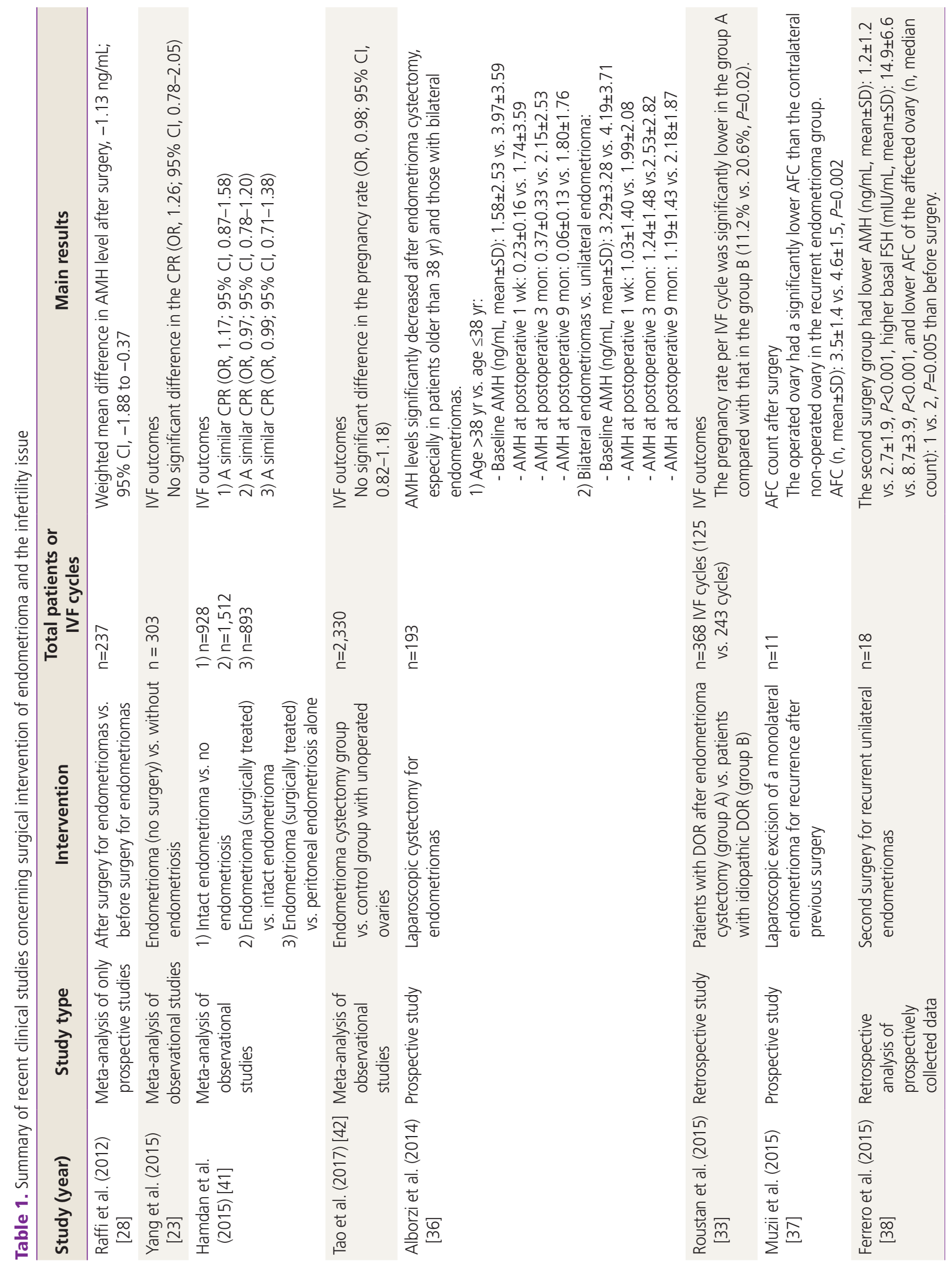




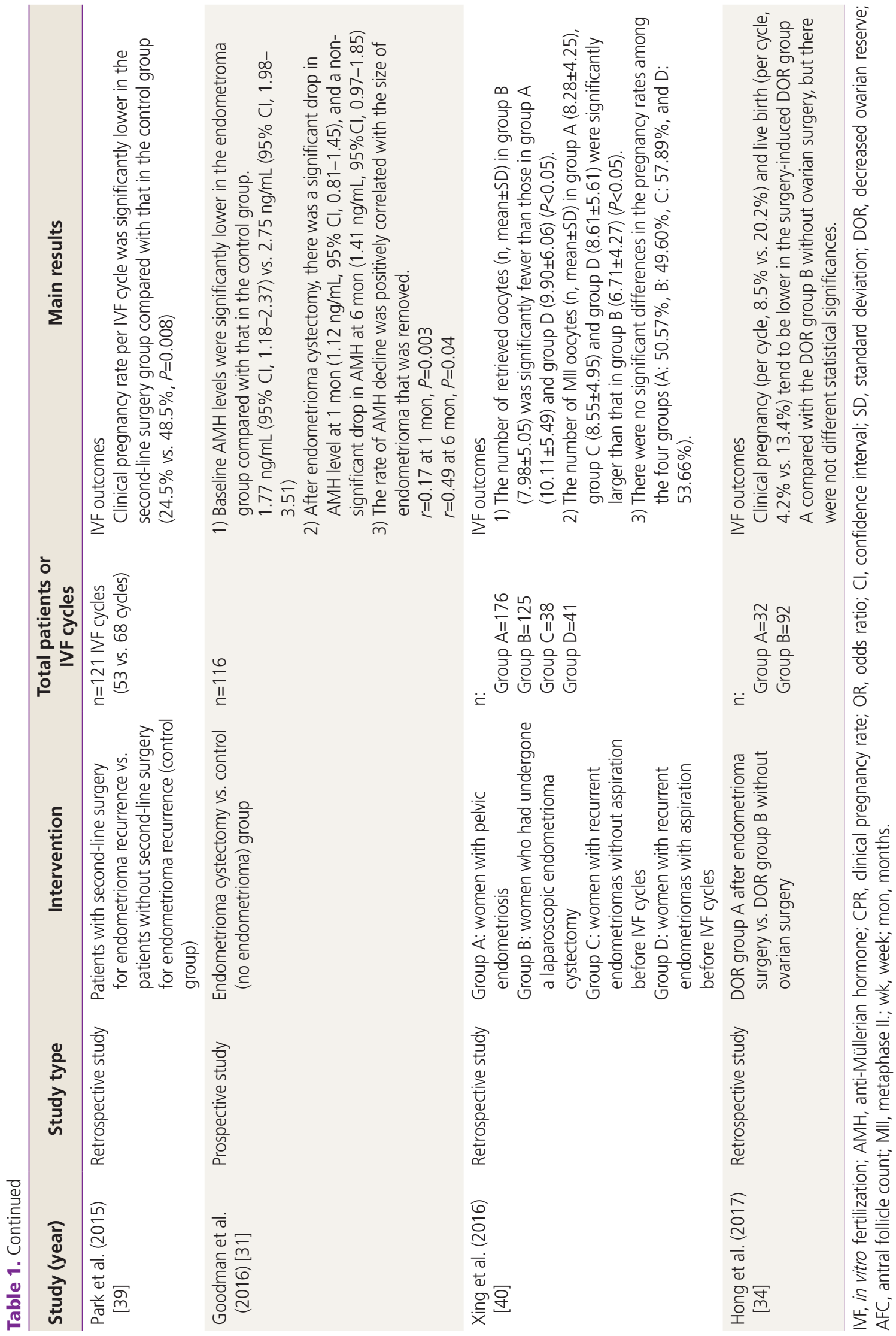




\section{Obstetrics \& Gynecology Science}

Hyun Jong Park, et al. Surgery and IVF in endometrioma

for 6 months prior to IVF (group II), and a patient group who had no hormonal therapy prior to IVF (group III). The pregnancy rates in groups I, II, and III were reported to be $44.7 \%$, $34.3 \%$, and $16.7 \%$, respectively. A significant difference in clinical pregnancy rate was found between groups I and III $(P=0.012)$. No significant difference was found between groups II and III. While long-term administration of the GnRH agonist conferred a risk of reduced endometrial receptivity, it was thought to show more positive results in terms of clinical pregnancy rates with concurrent dienogest use, as it could improve progesterone resistance in the endometrium [58].

If surgical intervention for endometriosis is performed, when would be the best time to perform ART after the surgery? A retrospective study on ART timing has recently been published. AlKudmani et al. [59] reported that the ongoing pregnancy rate was significantly higher when IVF was performed between 7 and 25 months after surgery for endometriosis than when IVF was performed between 0 and 3 months after surgery. Therefore, if IVF is planned after surgical intervention for endometriosis, administration of longterm hormone therapy prior to IVF should be considered for the suppression of residual endometriotic lesions, rather than performing IVF immediately after surgery to improve the pregnancy rate in IVF. Additional prospective studies about this topic should be conducted in the future.

\section{Summary}

Endometriosis could be toxic to the ovary and may reduce the ovarian reserve. Endometriosis may have negative effects on oocyte and embryo development. Clinically, the more advanced the endometriosis stage and the bigger the endometrioma, the poorer the reproductive outcomes of IVF. However, no clear evidence supports the use of surgical intervention before IVF to improve the reproductive outcomes of IVF in women with advanced-stage endometriosis or endometrioma. When the pain is severe or when cancer is suspected, laparoscopic ovarian cystectomy prior to IVF may be necessary and justified. When the size of the endometrioma is very large, surgery could be required prior to IVF to facilitate access to follicles during oocyte retrieval or to improve the ovarian response to controlled ovarian stimulation.

IVF as a means of achieving pregnancy could not overcome all the negative effects of endometriosis on fertility [60]. In infertile women with advanced-stage endometriosis or endometrioma, individualizing treatment methods for fertility is important. Basically, unconditional surgery prior to IVF was not recommended in these women. When the qualities of oocytes and embryos are observed to be seriously degraded in IVF cycles or when recurrent implantation fails occur in IVF cycles, surgical intervention for endometrioma could be considered. Restoration of a normal uterine anatomy by surgical intervention could help improve IVF outcomes in infertile women with severe endometriosis and repeated IVF failures [49]. In addition, prolonged pituitary downregulation prior to IVF cycles to suppress remnant postoperative lesions, may be helpful to increase the clinical pregnancy rate in subsequent IVF cycles. In the future, further prospective studies may be required to establish more-detailed guidelines for preserving the fertility of women with endometriosis.

\section{Conflict of interest}

No potential conflict of interest relevant to this article was reported.

\section{References}

1. Ozkan S, Murk W, Arici A. Endometriosis and infertility: epidemiology and evidence-based treatments. Ann N Y Acad Sci 2008;1127:92-100.

2. Centers for Disease Control and Prevention, American Society for Reproductive Medicine, Society for Assisted Reproductive Technology. 2013 assisted reproductive technology national summary report [Internet]. Atlanta (GA): US Department of Health and Human Services; 2015 [cited 2016 Dec 1]. Available from: http://www. cdc.gov/art/pdf/2013-report/art_2013_national_summary_report.pdf.

3. Singh SS, Suen MW. Surgery for endometriosis: beyond medical therapies. Fertil Steril 2017;107:549-54.

4. Dunselman GA, Vermeulen N, Becker C, Calhaz-Jorge C, D'Hooghe T, De Bie B, et al. ESHRE guideline: management of women with endometriosis. Hum Reprod 2014;29:400-12.

5. Marcoux S, Maheux R, Bérubé S; Canadian Collaborative Group on Endometriosis. Laparoscopic surgery in 


\section{Obstetrics \& Gynecology Science}

Vol. 62, No. 1, 2019

infertile women with minimal or mild endometriosis. N Engl J Med 1997;337:217-22.

6. Ruiz-Flores FJ, Garcia-Velasco JA. Is there a benefit for surgery in endometrioma-associated infertility? Curr Opin Obstet Gynecol 2012;24:136-40.

7. Garcia-Velasco JA, Somigliana E. Management of endometriomas in women requiring IVF: to touch or not to touch. Hum Reprod 2009;24:496-501.

8. American Society for Reproductive Medicine. Revised American Society for Reproductive Medicine classification of endometriosis: 1996. Fertil Steril 1997;67:81721.

9. lizuka M, Igarashi M, Abe Y, Ibuki Y, Koyasu Y, Ikuma K. Chemical assay of iron in ovarian cysts: a new diagnostic method to evaluate endometriotic cysts. Gynecol Obstet Invest 1998;46:58-60.

10. Yamaguchi K, Mandai M, Toyokuni S, Hamanishi J, Higuchi T, Takakura K, et al. Contents of endometriotic cysts, especially the high concentration of free iron, are a possible cause of carcinogenesis in the cysts through the iron-induced persistent oxidative stress. Clin Cancer Res 2008;14:32-40.

11. Iwabuchi T, Yoshimoto C, Shigetomi H, Kobayashi H. Oxidative stress and antioxidant defense in endometriosis and its malignant transformation. Oxid Med Cell Longev 2015;2015:848595.

12. Vercellini $P$, Crosignani $P$, Somigliana $E$, Viganò $P$, Buggio $L$, Bolis $G$, et al. The 'incessant menstruation' hypothesis: a mechanistic ovarian cancer model with implications for prevention. Hum Reprod 2011;26:2262-73.

13. Matsuzaki S, Schubert B. Oxidative stress status in normal ovarian cortex surrounding ovarian endometriosis. Fertil Steril 2010;93:2431-2.

14. Zhang X, Li XH, Ma X, Wang ZH, Lu S, Guo YL. Redoxinduced apoptosis of human oocytes in resting follicles in vitro. J Soc Gynecol Investig 2006;13:451-8.

15. Sanchez AM, Viganò $P$, Somigliana $E$, Panina-Bordignon $P$, Vercellini $P$, Candiani $M$. The distinguishing cellular and molecular features of the endometriotic ovarian cyst: from pathophysiology to the potential endometrioma-mediated damage to the ovary. Hum Reprod Update 2014;20:217-30.

16. Qiu JJ, Liu YL, Liu MH, Chen LP, Xu DW, Zhang ZX, et al. Ovarian interstitial blood flow changes assessed by transvaginal colour Doppler sonography: predicting ovarian endometrioid cyst-induced injury to ovarian interstitial vessels. Arch Gynecol Obstet 2012;285:427-33.

17. Simón C, Gutiérrez A, Vidal A, de los Santos MJ, Tarín JJ, Remohí J, et al. Outcome of patients with endometriosis in assisted reproduction: results from in-vitro fertilization and oocyte donation. Hum Reprod 1994;9:725-9.

18. Díaz I, Navarro J, Blasco L, Simón C, Pellicer A, Remohí J. Impact of stage III-IV endometriosis on recipients of sibling oocytes: matched case-control study. Fertil Steril 2000;74:31-4.

19. Shebl O, Sifferlinger I, Habelsberger A, Oppelt P, Mayer $R B$, Petek $E$, et al. Oocyte competence in in vitro fertilization and intracytoplasmic sperm injection patients suffering from endometriosis and its possible association with subsequent treatment outcome: a matched casecontrol study. Acta Obstet Gynecol Scand 2017;96:73644.

20. Barnhart K, Dunsmoor-Su R, Coutifaris C. Effect of endometriosis on in vitro fertilization. Fertil Steril 2002;77: 1148-55.

21. Harb HM, Gallos ID, Chu J, Harb M, Coomarasamy A. The effect of endometriosis on in vitro fertilisation outcome: a systematic review and meta-analysis. BJOG 2013;120:1308-20.

22. Hamdan M, Omar SZ, Dunselman G, Cheong Y. Influence of endometriosis on assisted reproductive technology outcomes: a systematic review and meta-analysis. Obstet Gynecol 2015;125:79-88.

23. Yang C, Geng Y, Li Y, Chen C, Gao Y. Impact of ovarian endometrioma on ovarian responsiveness and IVF: a systematic review and meta-analysis. Reprod Biomed Online 2015;31:9-19.

24. Romualdi D, Franco Zannoni G, Lanzone A, Selvaggi L, Tagliaferri V, Gaetano Vellone V, et al. Follicular loss in endoscopic surgery for ovarian endometriosis: quantitative and qualitative observations. Fertil Steril 2011;96:374-8.

25. Kim JY, Jee BC, Suh CS, Kim SH. Preoperative serum anti-mullerian hormone level in women with ovarian endometrioma and mature cystic teratoma. Yonsei Med J 2013;54:921-6.

26. Somigliana E, Berlanda N, Benaglia L, Viganò P, Vercellini $P$, Fedele L. Surgical excision of endometriomas and ovarian reserve: a systematic review on serum antimüllerian hormone level modifications. Fertil Steril 


\section{Obstetrics \& Gynecology Science}

Hyun Jong Park, et al. Surgery and IVF in endometrioma

2012;98:1531-8.

27. Ata B, Uncu G. Impact of endometriomas and their removal on ovarian reserve. Curr Opin Obstet Gynecol 2015;27:235-41.

28. Raffi F, Metwally M, Amer S. The impact of excision of ovarian endometrioma on ovarian reserve: a systematic review and meta-analysis. J Clin Endocrinol Metab 2012;97:3146-54.

29. Uncu G, Kasapoglu I, Ozerkan K, Seyhan A, Oral Yilmaztepe A, Ata B. Prospective assessment of the impact of endometriomas and their removal on ovarian reserve and determinants of the rate of decline in ovarian reserve. Hum Reprod 2013;28:2140-5.

30. Urman B, Alper E, Yakin K, Oktem O, Aksoy S, Alatas C, et al. Removal of unilateral endometriomas is associated with immediate and sustained reduction in ovarian reserve. Reprod Biomed Online 2013;27:212-6.

31. Goodman LR, Goldberg JM, Flyckt RL, Gupta M, Harwalker J, Falcone T. Effect of surgery on ovarian reserve in women with endometriomas, endometriosis and controls. Am J Obstet Gynecol 2016;215:589.e1-6.

32. Chen $Y$, Pei $H$, Chang $Y$, Chen $M$, Wang $H$, Xie $H$, et al. The impact of endometrioma and laparoscopic cystectomy on ovarian reserve and the exploration of related factors assessed by serum anti-Mullerian hormone: a prospective cohort study. J Ovarian Res 2014;7:108.

33. Roustan A, Perrin J, Debals-Gonthier M, PaulmyerLacroix O, Agostini A, Courbiere B. Surgical diminished ovarian reserve after endometrioma cystectomy versus idiopathic DOR: comparison of in vitro fertilization outcome. Hum Reprod 2015;30:840-7.

34. Hong SB, Lee NR, Kim SK, Kim H, Jee BC, Suh CS, et al. In vitro fertilization outcomes in women with surgery induced diminished ovarian reserve after endometrioma operation: comparison with diminished ovarian reserve without ovarian surgery. Obstet Gynecol Sci 2017;60:638.

35. Maignien C, Santulli P, Gayet V, Lafay-Pillet MC, Korb $D$, Bourdon $\mathrm{M}$, et al. Prognostic factors for assisted reproductive technology in women with endometriosisrelated infertility. Am J Obstet Gynecol 2017;216:280. e1-9.

36. Alborzi S, Keramati P, Younesi M, Samsami A, Dadras N. The impact of laparoscopic cystectomy on ovarian reserve in patients with unilateral and bilateral endome- triomas. Fertil Steril 2014;101:427-34.

37. Muzii L, Achilli C, Lecce F, Bianchi A, Franceschetti S, Marchetti $C$, et al. Second surgery for recurrent endometriomas is more harmful to healthy ovarian tissue and ovarian reserve than first surgery. Fertil Steril 2015;103:738-43.

38. Ferrero S, Scala C, Racca A, Calanni L, Remorgida V, Venturini $\mathrm{PL}$, et al. Second surgery for recurrent unilateral endometriomas and impact on ovarian reserve: a case-control study. Fertil Steril 2015;103:1236-43.

39. Park H, Kim CH, Kim EY, Moon JW, Kim SH, Chae HD, et al. Effect of second-line surgery on in vitro fertilization outcome in infertile women with ovarian endometrioma recurrence after primary conservative surgery for moderate to severe endometriosis. Obstet Gynecol Sci 2015;58:481-6.

40. Xing W, Lin H, Wu Z, Li Y, Zhang Q. Effect of pelvic endometriosis, endometriomas and recurrent endometriomas on IVF-ET/ICSI outcomes. Mater Sociomed 2016;28:91-4.

41. Hamdan M, Dunselman G, Li TC, Cheong Y. The impact of endometrioma on IVF/ICSI outcomes: a systematic review and meta-analysis. Hum Reprod Update 2015;21:809-25.

42. Tao X, Chen L, Ge S, Cai L. Weigh the pros and cons to ovarian reserve before stripping ovarian endometriomas prior to IVF/ICSI: a meta-analysis. PLoS One 2017;12:e0177426.

43. Hart RJ, Hickey M, Maouris P, Buckett W. Excisional surgery versus ablative surgery for ovarian endometriomata. Cochrane Database Syst Rev 2008:CD004992.

44. Benschop L, Farquhar C, van der Poel N, Heineman MJ. Interventions for women with endometrioma prior to assisted reproductive technology. Cochrane Database Syst Rev 2010:CD008571.

45. Somigliana E, Vercellini P, Viganó P, Ragni G, Crosignani PG. Should endometriomas be treated before IVF-ICSI cycles? Hum Reprod Update 2006;12:57-64.

46. Tanbo T, Fedorcsak P. Endometriosis-associated infertility: aspects of pathophysiological mechanisms and treatment options. Acta Obstet Gynecol Scand 2017;96:65967.

47. Giacomini E, Sanchez AM, Sarais V, Beitawi SA, Candiani $M$, Viganò $P$. Characteristics of follicular fluid in ovaries with endometriomas. Eur J Obstet Gynecol Reprod Biol 


\section{Obstetrics \& Gynecology Science}

Vol. 62, No. 1, 2019

2017;209:34-8.

48. Cooney LG, Mainigi MA. Surgery for endometriomas before IVF: what are the benefits and risks? Minerva Ginecol 2015;67:195-205.

49. Soriano D, Adler I, Bouaziz J, Zolti M, Eisenberg VH, Goldenberg $M$, et al. Fertility outcome of laparoscopic treatment in patients with severe endometriosis and repeated in vitro fertilization failures. Fertil Steril 2016;106:1264-9.

50. Reinblatt SL, Ishai L, Shehata F, Son WY, Tulandi T, Almog B. Effects of ovarian endometrioma on embryo quality. Fertil Steril 2011;95:2700-2.

51. Sallam HN, Garcia-Velasco JA, Dias S, Arici A. Long-term pituitary down-regulation before in vitro fertilization (IVF) for women with endometriosis. Cochrane Database Syst Rev 2006:CD004635.

52. Taketani Y, Kuo TM, Mizuno M. Comparison of cytokine levels and embryo toxicity in peritoneal fluid in infertile women with untreated or treated endometriosis. Am J Obstet Gynecol 1992;167:265-70.

53. Wu MY, Chen SU, Chao KH, Chen CD, Yang YS, Ho HN. Mouse embryo toxicity of IL-6 in peritoneal fluids from women with or without endometriosis. Acta Obstet Gynecol Scand 2001;80:7-11.

54. Gómez-Torres MJ, Acién P, Campos A, Velasco I. Embryotoxicity of peritoneal fluid in women with endometriosis. Its relation with cytokines and lymphocyte populations. Hum Reprod 2002;17:777-81.
55. Ruan HC, Zhu XM, Luo Q, Liu AX, Qian YL, Zhou CY, et al. Ovarian stimulation with $\mathrm{GnRH}$ agonist, but not $\mathrm{GnRH}$ antagonist, partially restores the expression of endometrial integrin $\beta 3$ and leukaemia-inhibitory factor and improves uterine receptivity in mice. Hum Reprod 2006;21:2521-9.

56. Strowitzki T, Faustmann T, Gerlinger C, Seitz C. Dienogest in the treatment of endometriosis-associated pelvic pain: a 12-week, randomized, double-blind, placebocontrolled study. Eur J Obstet Gynecol Reprod Biol 2010; 151:193-8.

57. Muller V, Kogan I, Yarmolinskaya M, Niauri D, Gzgzyan A, Aylamazyan E. Dienogest treatment after ovarian endometrioma removal in infertile women prior to IVF. Gynecol Endocrinol 2017;33:18-21.

58. Hayashi A, Tanabe A, Kawabe S, Hayashi M, Yuguchi H, Yamashita $Y$, et al. Dienogest increases the progesterone receptor isoform $\mathrm{B} / \mathrm{A}$ ratio in patients with ovarian endometriosis. J Ovarian Res 2012;5:31.

59. AlKudmani B, Gat I, Buell D, Salman J, Zohni K, Librach $C$, et al. In vitro fertilization success rates after surgically treated endometriosis and effect of time interval between surgery and in vitro fertilization. J Minim Invasive Gynecol 2018;25:99-104.

60. Somigliana E, Vigano P, Benaglia L, Busnelli A, Berlanda $\mathrm{N}$, Vercellini P. Management of endometriosis in the infertile patient. Semin Reprod Med 2017;35:31-7. 